FNAL-Pub-97/327-T

CERN-TH/97-190

IEM-FT-165/97

hep-ph/9710401

\title{
Electroweak Baryogenesis and Higgs and Stop Searches at LEP and the Tevatron 1
}

\author{
M. Carena ${ }^{a, b}$, M. Quirós ${ }^{c}$ and C.E.M. Wagner ${ }^{a}$ \\ ${ }^{a}$ CERN, TH Division, CH-1211 Geneva 23, Switzerland \\ ${ }^{b}$ Fermi National Accelerator Laboratory, P.O. Box 500, Batavia, IL 60510, U.S.A. \\ $\ddagger$ Instituto de Estructura de la Materia, CSIC, Serrano 123, 28006 Madrid, Spain
}

\begin{abstract}
It has been recently shown that the observed baryon number may originate at the electroweak phase transition, provided that the Higgs boson and the lightest stop are sufficiently light. In this work, we perform a detailed analysis, including all dominant two-loop finite temperature corrections to the Higgs effective potential, as well as the non-trivial effects proceeding from the mixing in the stop sector, to define the region of parameter space for which electroweak baryogenesis can happen. The limits on the stop and Higgs masses are obtained by taking into account the experimental bounds on these quantities, as well as those coming from the requirement of avoiding dangerous color breaking minima. We find for the Higgs mass $m_{h} \lesssim 105 \mathrm{GeV}$, while the stop mass may be close to the present experimental bound and must be smaller than, or of order of, the top quark mass. These results provide a very strong motivation for further non-perturbative analysis of the electroweak phase transition, as well as for the search for Higgs and stop particles at the LEP and Tevatron colliders.
\end{abstract}

October 1997

\footnotetext{
${ }^{*}$ Work supported in part by the European Union (contract CHRX/CT92-0004) and CICYT of Spain (contract AEN95-0195).
} 


\section{Introduction}

Recently, there has been renewed interest in the idea of generating the observed baryon number [1] at the electroweak phase transition [2]- [5]. A physical realization of this idea seems to be possible only by going beyond the Standard Model formulation. In fact, if no fundamental standard model singlet scalar field is present in the low energy effective theory, electroweak baryogenesis seems to be naturally induced only if the Higgs mass is sufficiently light, and if new light scalar particles, with strong couplings to the Higgs field are present in the theory [6]. Both effects tend to induce a strongly first order electroweak phase transition [0]-[9], which allows to preserve the generated baryon number. Moreover, new CP-violating phases, beyond those present in the Standard Model, seem to be necessary to fuel the baryon number generation [10.

Minimal low energy supersymmetric extensions of the Standard Model fulfill the three above mentioned requirements. A light CP-even Higgs naturally appears in these models. Indeed, an upper bound, of order $125 \mathrm{GeV}$ can generally be set on the lightest CP-even Higgs mass [12]-14]. The superpartners of the top quark, whose coupling to the Higgs is of order of the top quark Yukawa coupling, provide the additional scalars which help in inducing a relatively strong first order phase transition [15]-17]. For this to happen, the lightest stop should have a mass of order of, or smaller than, the top quark mass [18]-23]. Moreover, new CP-violating phases, induced by the supersymmetry breaking parameters associated with the left-right stop mixing, are naturally present in these theories [11]. A very recent analysis shows that an explanation of the observed baryon number of the universe requires CP-violating phases of order one and chargino and neutralino masses not much larger than $200 \mathrm{GeV}$ [24]. Similar results were obtained [25] by different analysis (see also Refs. [26, 27, 28]).

The above results present a strong case for the search of Higgs and top-squark particles at LEP and the Tevatron colliders [29]. It is therefore of the highest interest to define, in the most accurate possible way, the exact region of parameter space consistent with electroweak baryogenesis in the $m_{h}-m_{\widetilde{t}}$ plane. As has been first shown in Refs. [22, 23], two loop effects are very important to define the correct boundary of this region. In Ref. 30] it was suggested that, in the case of zero mixing in the stop sector, the phase transition can be sufficiently strong for Higgs masses as high as $100 \mathrm{GeV}$, if the stop mass is inside a narrow band, of order $150 \mathrm{GeV}$.

In this work, we define the allowed parameter space, including all dominant twoloop corrections to the Higgs effective potential and allowing a non-trivial mixing in the stop sector. We show that stop mixing effects highly increase the region of stop masses consistent with electroweak baryogenesis. Since light stops may induce color breaking minima, which should be taken into account in the analysis [18, the finite temperature effective potential along the squark and Higgs directions is computed. We identify the regions of parameter space in which the physical vacuum is stable within the thermal evolution of the universe, as well as those in which a two-step phase transition, via a color breaking minimum, can take place. Finally, we also define the region of parameter space in which the system is driven to the physical vacuum state at high temperatures, but this state becomes unstable at low temperatures. We define the boundaries which separate the regions consistent with the different above mentioned possibilities and show 
that, even in the case of absolute stability of the physical vacuum, stops as light as 100 $120 \mathrm{GeV}$ may be consistent with electroweak baryogenesis, for Higgs masses in the range 80-100 GeV.

In section 2 we summarize the most important properties of the effective potential at finite temperature. The expressions of the effective potentials for the stop and Higgs fields, with all dominant finite temperature two-loop corrections included, are given in appendix $\mathrm{A}$ and $\mathrm{B}$, respectively. In section 3 we define the different regions of parameter space to be studied in this article. In secion 4 we present our results. We reserve section 5 for our conclusions.

\section{Finite temperature effective potential}

In order to preserve the generated baryon number after the electroweak phase transition, a large vacuum expectation value of the Higgs field at the critical temperature is required [5]

$$
\frac{v\left(T_{c}\right)}{T_{c}} \gtrsim 1
$$

where $v=\langle\phi\rangle$ and $v(0) \simeq 246 \mathrm{GeV}$. The phase transition must be hence strongly first order. The implications of the above bound may only be obtained by a detailed knowledge of the electroweak phase transition. In the Standard Model, it has been shown that the two-loop improved effective potential leads to values of the order parameter and the critical temperature in good agreement with those obtained through a lattice non-perturbative analysis [7, 9, 31]. In this case the requirement of Eq. (2.1) demands a value of the Higgs mass which has been already ruled out by experimental searches. In this work, we shall present a two-loop perturbative analysis for the case of the Minimal Supersymmetric Standard Model (MSSM), in the expectation of a complete numerical simulation of this model.

Following the methods developed in Ref. [8], we compute the four dimensional finite temperature effective potential keeping all dominant two-loop finite temperature corrections induced by the Standard Model gauge bosons as well as by the light squarks and Higgs particles. As has been first shown in Ref. [22], the two-loop corrections to the Higgs effective potential in the Minimal Supersymmetric Model are dominated by loops involving the light stops and the $S U(3)$ gauge bosons, and strongly depend on the QCD coupling.

Although we reserve the technical details of the effective potential calculation for appendix A, we summarize here the most important properties of the two-loop finite temperature effective potential for the Higgs field. First of all, at one loop, the dominant corrections to the effective potential come from the gauge bosons, the top quark and its supersymmetric partners. We shall work in the limit in which the left handed stop is heavy, $m_{Q} \gtrsim 500 \mathrm{GeV}$. In this limit, the supersymmetric corrections to the precision electroweak parameter $\Delta \rho$ become small and hence, this allows a good fit to the electroweak precision data coming from LEP and SLD I. Lower values of $m_{Q}$ make

\footnotetext{
${ }^{1} \mathrm{~A}$ lighter left-handed stop may also be consistent with precision electroweak measurements [32]. However, this requires sizeable values of the stop mixing mass parameter, which tend to suppress the
} 
the phase transition stronger and we are hence taking a conservative assumption from the point of view of defining the region consistent with electroweak baryogenesis. The left handed stop decouples at finite temperature, but, at zero temperature, it sets the scale of the Higgs masses as a function of $\tan \beta$. For right-handed stop masses below, or of order of, the top quark mass, and for large values of the CP-odd Higgs mass, $m_{A} \gg M_{Z}$, the one-loop improved Higgs effective potential admits a high temperature expansion,

$V_{0}(\phi)+V_{1}(\phi, T)=-\frac{m^{2}(T)}{2} \phi^{2}-T\left[E_{\mathrm{SM}} \phi^{3}+\left(2 N_{c}\right) \frac{\left(m_{\widetilde{t}}^{2}+\Pi_{\widetilde{t}_{R}}(T)\right)^{3 / 2}}{12 \pi}\right]+\frac{\lambda(T)}{8} \phi^{4}+\cdots$

where

$$
\Pi_{\widetilde{t}_{R}}(T)=\frac{4}{9} g_{s}^{2} T^{2}+\frac{1}{6} h_{t}^{2}\left[1+\sin ^{2} \beta\left(1-\widetilde{A}_{t}^{2} / m_{Q}^{2}\right)\right] T^{2}+\left(\frac{1}{3}-\frac{1}{18}|\cos 2 \beta|\right) g^{2} T^{2}
$$

is the finite temperature self-energy contribution to the right-handed squarks (see appendix A), $g_{s}$ is the strong gauge coupling, $N_{c}=3$ is the number of colours and

$$
E_{\mathrm{SM}} \sim \frac{1}{3}\left(\frac{2 M_{W}^{3}+M_{Z}^{3}}{2 \pi v^{3}}\right)
$$

is the cubic term coefficient in the Standard Model case.

Within our approximation, the lightest stop mass is approximately given by

$$
m_{\widetilde{t}}^{2} \simeq m_{U}^{2}+0.15 M_{Z}^{2} \cos 2 \beta+m_{t}^{2}\left(1-\frac{\tilde{A}_{t}^{2}}{m_{Q}^{2}}\right)
$$

where $\tilde{A}_{t}=A_{t}-\mu / \tan \beta$ is the stop mixing parameter and $m_{t}=h_{t} \phi \sin \beta / \sqrt{2}$. As was observed in Ref. [18], the phase transition strength is maximized for values of the soft breaking parameter $m_{U}^{2}=-\Pi_{\widetilde{t}_{R}}(T)$, for which the coefficient of the cubic term in the effective potential,

$$
E \simeq E_{\mathrm{SM}}+\frac{h_{t}^{3} \sin ^{3} \beta\left(1-\widetilde{A}_{t}^{2} / m_{Q}^{2}\right)^{3 / 2}}{4 \sqrt{2} \pi}
$$

which governs the strength of the phase transition,

$$
\frac{v\left(T_{c}\right)}{T_{c}} \simeq \frac{4 E}{\lambda}
$$

can be one order of magnitude larger than $E_{\mathrm{SM}}$ [18]. In principle, the above would allow a sufficiently strong first order phase transition for Higgs masses as large as 100 $\mathrm{GeV}$. However, it was also noticed that such large negative values of $m_{U}^{2}$ may induce the presence of color breaking minima at zero or finite temperature [34, 18]. Demanding the absence of such dangerous minima, the one loop analysis leads to an upper bound on

phase transition strength. 
the lightest CP-even Higgs mass of order $80 \mathrm{GeV}$. This bound was obtained for values of $\widetilde{m}_{U}^{2}=-m_{U}^{2}$ of order $(80 \mathrm{GeV})^{2}$.

The most important two loop corrections are of the form $\phi^{2} \log (\phi)$ and, as said above, are induced by the Standard Model weak gauge bosons as well as by the stop and gluon loops [8, 22]. It was recently noticed that the coefficient of these terms can be efficiently obtained by the study of the three dimensional running mass of the scalar top and Higgs fields in the dimensionally reduced theory at high temperatures [30]. Equivalently, in a four dimensional computation of the MSSM Higgs effective potential with a heavy left-handed stop, we obtain

$$
V_{2}(\phi, T) \simeq \frac{\phi^{2} T^{2}}{32 \pi^{2}}\left[\frac{51}{16} g^{2}-3\left[h_{t}^{2} \sin \beta^{2}\left(1-\frac{\widetilde{A}_{t}^{2}}{m_{Q}^{2}}\right)\right]^{2}+8 g_{s}^{2} h_{t}^{2} \sin ^{2} \beta\left(1-\frac{\widetilde{A}_{t}^{2}}{m_{Q}^{2}}\right)\right] \log \left(\frac{\Lambda_{H}}{\phi}\right)
$$

where the first term comes from the Standard Model gauge boson-loop contributions, while the second and third terms come from the light supersymmetric particle loop contributions. The scale $\Lambda_{H}$ depends on the finite corrections, which may be obtained by the expressions given in appendix A 2. As mentioned above, the two-loop corrections are very important and, as has been shown in Ref. [22], they can make the phase transition strongly first order even for $m_{U} \simeq 0$.

An analogous situation occurs in the $U$-direction. The one-loop expression is approximately given by

$$
V_{0}(U)+V_{1}(U, T)=\left(-\widetilde{m}_{U}^{2}+\gamma_{U} T^{2}\right) U^{2}-T E_{U} U^{3}+\frac{\lambda_{U}}{2} U^{4}
$$

where

$$
\begin{aligned}
\gamma_{U} & \equiv \frac{\Pi_{\widetilde{t}_{R}}(T)}{T^{2}} \simeq \frac{4 g_{s}^{2}}{9}+\frac{h_{t}^{2}}{6}\left[1+\sin ^{2} \beta\left(1-\widetilde{A}_{t}^{2} / m_{Q}^{2}\right)\right] ; \quad \lambda_{U} \simeq \frac{g_{s}^{2}}{3} \\
E_{U} & \simeq\left[\frac{\sqrt{2} g_{s}^{2}}{6 \pi}\left(1+\frac{2}{3 \sqrt{3}}\right)\right] \\
& +\left\{\frac{g_{s}^{3}}{12 \pi}\left(\frac{5}{3 \sqrt{3}}+1\right)+\frac{h_{t}^{3} \sin ^{3} \beta\left(1-\widetilde{A}_{t}^{2} / m_{Q}^{2}\right)^{3 / 2}}{3 \pi}\right\} .
\end{aligned}
$$

The contribution to $E_{U}$ inside the squared brackets comes from the transverse gluons, while the one inside the curly brackets comes from the squark and Higgs contributions 18.

Analogous to the case of the field $\phi$, the two loop corrections to the $U$-potential are dominated by gluon and stop loops and are approximately given by

$$
V_{2}(U, T)=\frac{U^{2} T^{2}}{16 \pi^{2}}\left[\frac{100}{9} g_{s}^{4}-2 h_{t}^{2} \sin ^{2} \beta\left(1-\frac{\widetilde{A}_{t}^{2}}{m_{Q}^{2}}\right)\right] \log \left(\frac{\Lambda_{U}}{U}\right)
$$

where, as in the Higgs case, the scale $\Lambda_{U}$ may only be obtained after the finite corrections to the effective potential, given in appendix B, are computed.

\footnotetext{
${ }^{2}$ In the numerical computations, we use the whole expression given in appendix A.
} 
Once the effective potential in the $\phi$ and $U$ directions are computed, one can study the strength of the electroweak phase transition, as well as the presence of potential color breaking minima (see section 3). At one-loop, it was observed that requiring the stability of the physical vacuum at zero temperature was enough to assure the absolute stability of the potential at finite temperature. As has been first noticed in Ref. [30], once two loop corrections are included, the situation is more complicated. We shall proceed with this analysis in the next section.

\section{Color breaking minima}

As explained above, we work in the limit of large values of $m_{Q}$, for which small contributions to $\Delta \rho$ are expected. As has been shown in Ref. [18, in this limit a color breaking minimum with $\langle Q\rangle \neq 0$ does not develop at $T=0$, unless the stop mixing parameter $\widetilde{A}_{t}$ is very large. These large values of $\widetilde{A}_{t}$ suppress the strength of the first order phase transition and are hence of no interest for this study. We expect no modification of this conclusion at $T \neq 0$, so far as the relation $m_{Q} \gg T$ is preserved.

At zero temperature, for $Q=0$ the minimization of the effective potential for the fields $\phi$ and $U$ shows that the true minima are located for vanishing values of one of the two fields. The two set of minima are connected through a family of saddle points for which both fields acquire non-vanishing values. Due to the nature of the high temperature corrections, we do not expect a modification of this conclusion at finite temperature. A detailed analysis of this problem would however be needed to decide whether minima with $\phi \neq 0$ and $U \neq 0$ for $Q=0$ exist at finite temperature. We shall restrict our analysis to the behaviour of the electroweak and color breaking phase transitions in the directions $\phi \neq 0, U=0$ and $U \neq 0$ and $\phi=0$, respectively.

Two parameters control the presence of color breaking minima: $\widetilde{m}_{U}^{c}$, defined as the smallest value of $\widetilde{m}_{U}$ for which a color breaking minimum deeper than the electroweak breaking minimum is present at $T=0$, and $T_{c}^{U}$, the critical temperature for the transition into a color breaking minimum in the $U$-direction. The value of $\widetilde{m}_{U}^{c}$ may be obtained by analysing the effective potential for the field $U$ at zero temperature, and it is approximately given by [34, 18]

$$
\widetilde{m}_{U}^{c} \simeq\left(\frac{m_{H}^{2} v^{2} g_{s}^{2}}{12}\right)^{1 / 4}
$$

Defining the critical temperature as that at which the potential at the symmetry preserving and broken minima are degenerate, four situations can happen in the comparison of the critical temperatures along the $\phi\left(T_{c}\right)$ and $U\left(T_{c}^{U}\right)$ transitions:
a) $T_{c}^{U}<T_{c} ; \widetilde{m}_{U}<\widetilde{m}_{U}^{c}$
b) $T_{c}^{U}<T_{c} ; \widetilde{m}_{U}>\widetilde{m}_{U}^{c}$
c) $T_{c}^{U}>T_{c} ; \widetilde{m}_{U}<\widetilde{m}_{U}^{c}$
d) $T_{c}^{U}>T_{c} ; \widetilde{m}_{U}>\widetilde{m}_{U}^{c}$ 
In case a), as the universe cools down, a phase transition into a color preserving minimum occurs, which remains stable until $T=0$. This situation, of absolute stability of the physical vacuum, is the most conservative requirement to obtain electroweak baryogenesis. We shall generally demand these conditions to define the allowed parameter space in the $m_{h}-m_{\widetilde{t}}$ plane. In case b), at $T=0$ the color breaking minimum is deeper than the physical one implying that the color preserving minimum becomes unstable for finite values of the temperature, with $T<T_{c}$. A physically acceptable situation may only occur if the lifetime of the physical vacuum is smaller than the age of the universe. In the following, we shall denote this situation as "metastability". In case c), as the universe cools down, a color breaking minimum develops which, however, becomes metastable as the temperature approaches zero. A physically acceptable situation can only take place if a two step phase transition occurs, that is if the color breaking minimum has a lifetime lower than the age of the universe at some temperature $T<T_{c}$ [30]. Finally, in case d) the color breaking minimum is absolutely stable and hence, the situation becomes physically unacceptable.

As said before, at one-loop, the condition of having a color preserving minimum at zero temperature, $\widetilde{m}_{U}<\widetilde{m}_{U}^{c}$, automatically ensures that $T_{c}^{U}<T_{c}$. Hence, case c) never occurs and a two step phase transition is not allowed. On the contrary, as we shall show below, when two-loop corrections to the effective potential of the Higgs and stop fields are included, cases b) or c) may occur depending mainly on the values of the mixing in the stop sector and on $\tan \beta$.

Figure 1 shows the region of parameter space consistent with a sufficiently strong phase transition for four different values of the stop mixing $\widetilde{A}_{t}$. At the left of the solid line, $v / T \gtrsim 1$. Hence, for a given value of $\widetilde{A}_{t}$ and $m_{h}$, the solid line gives the upper bound on the stop mass consistent with the preservation of the generated baryon number at the electroweak phase transition. Since $v / T$ is inversely proportional to $m_{h}^{2}$, lower values of $m_{\widetilde{t}}$ (larger values of $E$ ) are needed as the Higgs mass is increased. The dashed line represents the values for which the critical temperature of the $U$ field is equal to the critical temperature for the electroweak phase transition. This means that $T_{c}>T_{c}^{U}$ $\left(T_{c}<T_{c}^{U}\right)$ on the right (left) of the dashed lines. Similarly, the short-dashed line is defined by the values of the Higgs and stop mass parameters for which $\widetilde{m}_{U}=\widetilde{m}_{U}^{c}$, i.e. $\widetilde{m}_{U}<\widetilde{m}_{U}^{c}\left(\widetilde{m}_{U}>\widetilde{m}_{U}^{c}\right)$ on the right (left) of the short-dashed lines. Observe that, for the same value of the stop mass parameters, larger Higgs masses are associated with larger values of $\tan \beta$.

From Fig. 1, it is clear that for low values of the mixing, $\widetilde{A}_{t} \lesssim 200 \mathrm{GeV}$, case a) or c) may occur but, contrary to what happens at one-loop, case b) is not realized. For the case of no mixing, this result is in agreement with the analysis of [30. The region of absolute stability of the physical vacuum for $\widetilde{A}_{t} \simeq 0$ is bounded to values of the Higgs mass of order $95 \mathrm{GeV}$. There is a small region at the right of the solid line, in which a two-step phase transition may take place, for values of the parameters which would lead to $v / T<1$ for $T=T_{c}$, but may evolve to larger values at some $T<T_{c}$ at which the second of the two step phase transition into the physical vacuum takes place. This region disappears for larger values of the stop mixing mass parameter. Quite generally, depending on the value of $\widetilde{A}_{t}$, the allowed region can be larger if a two step phase transition is considered, although the upper bound on the Higgs mass is not 
modified (see section 4). For values of the mixing parameter $\widetilde{A}_{t}$ between $200 \mathrm{GeV}$ and $300 \mathrm{GeV}$, both situations, cases b) and c) may occur, depending on the value of $\tan \beta$. For large values of the stop mixing, $\widetilde{A}_{t}>300 \mathrm{GeV}$, a two-step phase transition does not take place. The region of parameter space located at the left of both the dashed and short-dashed lines in the figure, corresponds to the case d) and hence is ruled out.

\section{Higgs and Stop mass constraints}

To define the allowed region of space in the $m_{h}-m_{\widetilde{t}}$ plane, one has to join all the allowed regions for different values of $\widetilde{A}_{t}$. As the value of $\widetilde{A}_{t}$ increases, the stop mass values tend to decrease, and hence the lower bound on the stop mass is usually obtained for larger values of $\widetilde{A}_{t}$. Since for larger values of $\widetilde{A}_{t}$, the phase transition becomes weaker, large values of the Higgs mass may only be obtained for low values of $\widetilde{A}_{t}$. If we demand absolute stability, the upper bound on $m_{h}$ decreases quite fast with $\widetilde{A}_{t}$, and the Higgs mass is constrained to be below $105,95,85 \mathrm{GeV}$ for $\widetilde{A}_{t}=300,500,600 \mathrm{GeV}$, respectively. Observe that, as becomes clear from Fig. 1, the same point in the $m_{h}-m_{\tilde{t}}$ plane may correspond to different values of the Higgs and stop parameters and hence could lead to different physical situations.

Figure 2 shows the allowed region of parameter space in the $m_{h}-m_{\widetilde{t}}$ plane. The solid line demarks the region where $v / T \gtrsim 1$ and the physical vacuum is absolutely stable. The right handed boundary is defined by the points of $v / T=1$ and $\widetilde{A}_{t} \simeq 0$ (values of $\widetilde{A}_{t} \gtrsim 100 \mathrm{GeV}$ are necessary to reach the largest values of $m_{h}$, see Fig. 1 ). The tiny region of parameter space, shown in the upper left panel of Fig. 1, for which a two step phase transition may occur for values of the stop mass at the right of the solid line, $v\left(T_{c}\right) / T_{c}<1$, is not shown in the figure. Observe that the uppermost values of $m_{h}$ are approximately constant, independent of $m_{\widetilde{t}}$ for a large region of parameter space. What constrains the Higgs mass in the region of $\widetilde{A}_{t} \lesssim 300 \mathrm{GeV}$ is not the mechanism of electroweak baryogenesis, but just the fact that, for the given values of $\widetilde{A}_{t}$ and $m_{Q}=1 \mathrm{TeV}$, the Higgs mass cannot reach a larger bound. Slightly stronger (weaker) constraints would be obtained for $m_{Q}=500 \mathrm{GeV}(2 \mathrm{TeV})$. Finally, the left boundary is defined by values of $\widetilde{m}_{U}=\widetilde{m}_{U}^{c}$, for $\widetilde{A}_{t} \gtrsim 300 \mathrm{GeV}$. As said before, for this range of values of the stop mixing, and as happens at the one-loop level, the condition of stability at zero temperature ensures the stability at finite temperature, as it becomes clear by observing the evolution of the different regions in Fig. 1.

All together, and even demanding absolute stability of the physical vacuum, electroweak baryogenesis seems to work for a wide region of Higgs and stop mass values. Higgs masses between the present experimental limit, of about $75 \mathrm{GeV}$ [36], and around $105 \mathrm{GeV}$ are consistent with this scenario. Similarly, the running stop mass may vary from values of order $165 \mathrm{GeV}$ (of the same order as the top quark mass one) and 100 $\mathrm{GeV}$. Observe that, due to the influence of the D-terms, values of $m_{\widetilde{t}} \simeq 165 \mathrm{GeV}, \widetilde{A}_{t} \simeq 0$ and $m_{h} \simeq 75 \mathrm{GeV}$, are achieved for small positive values of $m_{U}$ (of order $40 \mathrm{GeV}$ for the case represented in Fig. 2). Also observe that for lower values of $m_{Q}$ the phase transition may become more strongly first order and slightly larger values of the stop masses may be obtained. 
The region bounded by the thin dashed lines in Fig. 2 corresponds to values of $m_{h}$ $m_{\widetilde{t}}$ for which a two step phase transition can take place. Even though the effect of stop mixing greatly enlarges this region, we obtain from the present study that a two step phase transition may occur only in a narrow region of stop masses. The exact location of this band depends slightly on the value of $m_{Q}$. For lower values of $m_{Q}$, it moves to larger values of $m_{\tilde{t}}$. Finally, the allowed parameter space may be greatly increased if we consider the condition of metastability. However, to analyse whether this may lead to a physically acceptable situation, the lifetime of the finite temperature metastable vacuum should be computed, something which is beyond the scope of this article.

\section{Conclusions}

In this article, we have computed the region of stop and Higgs masses consistent with the preservation of the baryon number generated at the electroweak phase transition. We showed that, once two loop corrections are consistently included in both the Higgs and the stop effective potentials, the electroweak phase transition may occur at one or two steps, with the additional possibility of a metastable physical vacuum state. Demanding the condition of absolute stability of the physical vacuum at all temperatures, we find that the phase transition may be sufficiently strong if the Higgs mass $75 \mathrm{GeV} \lesssim m_{h} \lesssim$ $105 \mathrm{GeV}$ and the lightest stop mass $100 \mathrm{GeV} \lesssim m_{\widetilde{t}} \lesssim m_{t}$. The allowed $m_{\widetilde{t}} m_{h}$ region may be enlarged if the condition of metastability of the physical vacuum is considered, with stop mass parameters which are somewhat lower than in the case of an absolutely stable vacuum. A two step phase transition may only occur for values of the Higgs and stop masses which are also consistent with absolute stability, although the necessary stop mass parameters are slightly different.

Our results present a strong case for Higgs and stop searches at LEP and the Tevatron colliders. Indeed, by the end of 1999, LEP should be able to explore Higgs masses as high as $100 \mathrm{GeV}$, testing almost the whole region consistent with electroweak baryogenesis. An upgrade in energy, up to $\sqrt{s} \simeq 200 \mathrm{GeV}$ during the years 1999-2000 will allow to explore the remaining region. If the Higgs is found at LEP, the Tevatron shall be able to test a wide region of the allowed stop masses during the next run, to start at the end of 1999. The stop mass reach of the upgraded Tevatron depends on the stop decay channels, but the whole region of stop masses consistent with this scenario will be explored if the ultimate upgraded Tevatron (TeV33) becomes operative. Observe that, LEP is beginning to test the lowest values of the stop mass [36], which are compatible with a metastable electroweak vacuum state.

Limits on the stop and Higgs mass parameters can also be obtained from electroweak precision measurements and rare decay processes. As explained in section 2, the parameter $\Delta \rho$ sets a lower bound on the left handed stop mass. Another important measurement is the branching ratio $\operatorname{BR}(b \rightarrow s \gamma)$. Indeed, it has been recently argued 24] that the baryon number generation at the electroweak phase transition is suppressed for large values of the CP-odd Higgs mass p. Lower values of the CP-odd

\footnotetext{
${ }^{3}$ Observe, however, that both the Higgs mass and the value of $v\left(T_{c}\right) / T_{c}$ vary only slightly with $m_{A}$, for values of $m_{A} \gtrsim 250 \mathrm{GeV}$. The results presented in this article are hence expected to remain valid
} 
Higgs mass, and hence of the charged Higgs mass, tend to enhance the branching ratio of this rare decay with respect to the Standard Model value [37]. Since the Standard Model prediction for $\mathrm{BR}(b \rightarrow s \gamma)$ [38] is more than one standard deviation above the experimental value [39], a consistent result may only be obtained if the stop-chargino contributions partially cancel the charged Higgs induced enhancement. For the values of the parameters presented in Figure 1, and for a relatively light charged Higgs, $m_{H^{+}} \simeq 300 \mathrm{GeV}$ (and hence $m_{A} \simeq 300 \mathrm{GeV}$ ), this can only be achieved for moderate values of the stop mixing parameter $\widetilde{A}_{t}$ (see ref. [29]). The precise bound depends on the value of $\tan \beta, \mu$ and the charged Higgs mass, but values of $\widetilde{A}_{t} \lesssim 200 \mathrm{GeV}$ tend to be disfavored. Although this fact does not modify the upper bound on the Higgs mass, it shows that the largest values of the stop masses, obtained for low values of $\widetilde{A}_{t}$ tend to be disfavored (see Fig. 1).

Finally, it is important to stress that, since the above results are obtained by using a two-loop finite temperature effective potential method, a non-perturbative analysis will be necessary to support these calculations, providing a definite check of the region of parameter space consistent with electroweak baryogenesis.

\section{Acknowledgements}

M.C. and C.W. would like to thank the Aspen Center for Physics, where part of this work has been done. We would like to thank M. Shaposhnikov, M. Laine and M. Schmidt for very interesting discussions.

\section{Appendix A}

In this appendix we present some technical details concerning the effective potential at finite temperature along the $\phi$-direction, including the leading two-loop corrections. We will consider the case where the pseudoscalar mass $m_{A}$ is much larger than the temperature $T$. In this case only the light $C P$-even Higgs is coupled to the thermal bath, while the thermal distribution functions of the heavy neutral $C P$-even, the neutral $C P$ odd and the charged Higgses are Boltzmann suppressed and decouple from the thermal bath. We will work in the 't Hooft-Landau gauge and in the $\overline{\mathrm{MS}}$-renormalization scheme.

We will follow the notation and conventions of Ref. [35]. Dimensional regularization $(D=4-2 \epsilon)$ is used to evaluate divergent integrals. Poles in $1 / \epsilon$ and terms depending on $\iota_{\epsilon}$ will be dropped since they are canceled by counterterms [35]. We will fix the $\overline{\mathrm{MS}}$-scale $\bar{\mu}$ to the temperature $T$, and hence put to zero all terms in $\ln (\bar{\mu} / T)$, and all the couplings and fields will be considered at the scale $\bar{\mu}=T: g(T), \phi(T), \cdots$.

The states that give the most important contributions to the effective potential are the SM-particles: electroweak gauge bosons, the top quark, the Higgs and Goldstone bosons ( $W, Z, \gamma, t, h, \chi$, respectively), as well as the left and right handed (third generation) scalar tops $\left(\widetilde{t}_{L}, \widetilde{t}_{R}\right)$. The SM fields have tree-level masses:

$$
m_{W}^{2}=\frac{1}{4} g^{2} \phi^{2}
$$

for these values of $m_{A}$. 


$$
\begin{aligned}
m_{Z}^{2} & =\frac{1}{4}\left(g^{2}+g^{\prime 2}\right) \phi^{2} \\
m_{t}^{2} & =\frac{1}{2} \sin \beta^{2} h_{t}^{2} \phi^{2} \\
m_{h}^{2} & =\frac{1}{8}\left(g^{2}+g^{\prime 2}\right) \cos ^{2} 2 \beta\left(3 \phi^{2}-v^{2}\right) \\
m_{\chi}^{2} & =\frac{1}{8}\left(g^{2}+g^{\prime 2}\right) \cos ^{2} 2 \beta\left(\phi^{2}-v^{2}\right)
\end{aligned}
$$

and degrees of freedom (longitudinal, $L$, and transversal, $T$, for the gauge bosons)

$$
\begin{array}{rlrl}
n_{W_{L}} & =2 & & n_{W_{T}}=4 \\
n_{Z_{L}} & =1 & & n_{Z_{T}}=2 \\
n_{\gamma_{L}} & =1 & & n_{\gamma_{T}}=2 \\
n_{h} & =1 & & n_{\chi}=3 \\
n_{t} & =-12 .
\end{array}
$$

The stop fields $\left(\widetilde{t}_{L}, \widetilde{t}_{R}\right)$, with degrees of freedom,

$$
n_{\widetilde{t}_{L}}=n_{\widetilde{t}_{R}}=6
$$

have a squared mass matrix given by:

$$
\mathcal{M}_{\widetilde{t}}^{2}=\left(\begin{array}{cc}
m_{Q}^{2}+m_{t}^{2}+\frac{1}{8}\left(g^{2}-g^{\prime 2}\right) \cos 2 \beta \phi^{2} & m_{t} \widetilde{A}_{t} \\
m_{t} \widetilde{A}_{t} & m_{U}^{2}+m_{t}^{2}+\frac{1}{6} g^{\prime 2} \cos 2 \beta \phi^{2}
\end{array}\right)
$$

where the $L-R$ mixing is defined as

$$
\widetilde{A}_{t}=A_{t}-\mu / \tan \beta .
$$

The matrix (A.4) is diagonalized by the angle $\theta_{t}$ defined by

$$
\sin 2 \theta_{t}=\frac{2 \mathcal{M}_{12}^{2}}{\sqrt{\left(\operatorname{Tr} \mathcal{M}^{2}\right)^{2}-4 \operatorname{det} \mathcal{M}^{2}}} \quad \cos 2 \theta_{t}=\frac{\mathcal{M}_{11}^{2}-\mathcal{M}_{22}^{2}}{\sqrt{\left(\operatorname{Tr} \mathcal{M}^{2}\right)^{2}-4 \operatorname{det} \mathcal{M}^{2}}}
$$

and we will call $\widetilde{t}$ and $\widetilde{T}$ the light and heavy eigenstates, and $m_{\widetilde{t}}$ and $m_{\widetilde{T}}$ the corresponding eigenvalues.

We now define thermal masses for the longitudinal components of gauge bosons $\left(W_{L}, Z_{L}, \gamma_{L}\right)$ and for all scalars $\left(h, \chi, \tilde{t}_{L}, \widetilde{t}_{R}\right)$ by means of the corresponding selfenergies. We include in the thermal bath the SM particles ( $W, Z, \gamma, t, h$ and $\chi$ ), as well as the light stop $\widetilde{t}$, which plays a prominent role in the strength of the phase transition, and (light) charginos and neutralinos, which were proven to be essential for the generation of the baryon asymmetry [24]. The self-energies, to leading order, are given by:

$$
\Pi_{W}=\frac{7}{3} g^{2} T^{2}
$$




$$
\begin{aligned}
\Pi_{B} & =\frac{22}{9} g^{\prime 2} T^{2} \\
\Pi_{h} & =\frac{1}{16}\left(g^{2}+g^{\prime 2}\right) \cos ^{2} 2 \beta T^{2}+\frac{5}{16} g^{2} T^{2}+\frac{5}{48} g^{2} T^{2}+\frac{1}{2} h_{t}^{2} \sin ^{2} \beta T^{2} \\
\Pi_{\chi} & =\Pi_{h} \\
\Pi_{\widetilde{t}_{L}} & =\frac{1}{3} g_{s}^{2} T^{2}+\frac{5}{16} g^{2} T^{2}-\frac{1}{72} g^{\prime 2}\left(\frac{7}{6}+\cos 2 \beta\right) T^{2}+\frac{1}{12} h_{t}^{2}\left(2+\sin ^{2} \beta\right) T^{2} \\
\Pi_{\widetilde{t}_{R}} & =\frac{4}{9} g_{s}^{2} T^{2}+\frac{1}{18}(6+\cos 2 \beta) g^{\prime 2} T^{2}+\frac{1}{6} h_{t}^{2}\left[1+\sin ^{2} \beta\left(1-\frac{\widetilde{A}_{t}^{2}}{m_{Q}^{2}}\right)\right] T^{2} .
\end{aligned}
$$

The thermal masses are defined, for the SM bosons, as:

$$
\begin{aligned}
\bar{m}_{W_{L}}^{2} & =m_{W}^{2}+\Pi_{W} \\
\bar{m}_{Z_{L}, \gamma_{L}}^{2} & =\frac{1}{2}\left[\frac{1}{4}\left(g^{2}+g^{\prime 2}\right) \phi^{2}+\Pi_{W}+\Pi_{B}\right. \\
& \left. \pm \sqrt{\left(\left(g^{2}-g^{\prime 2}\right) \frac{\phi^{2}}{4}+\Pi_{W}-\Pi_{B}\right)^{2}+\frac{1}{4} g^{2} g^{\prime 2} \phi^{4}}\right] \\
\bar{m}_{h}^{2} & =m_{h}^{2}+\Pi_{h} \\
\bar{m}_{\chi}^{2} & =m_{\chi}^{2}+\Pi_{\chi}
\end{aligned}
$$

and for the stops as:

$\overline{\mathcal{M}}_{\widetilde{t}}^{2}=\left(\begin{array}{cc}m_{Q}^{2}+m_{t}^{2}+\frac{1}{8}\left(g^{2}-g^{2}\right) \cos 2 \beta \phi^{2}+\Pi_{\widetilde{t}_{L}} & m_{t} \widetilde{A}_{t} \\ m_{t} \widetilde{A}_{t} & m_{U}^{2}+m_{t}^{2}+\frac{1}{6} g^{\prime 2} \cos 2 \beta \phi^{2}+\Pi_{\widetilde{t}_{R}}\end{array}\right)$.

Similarly one can diagonalize the mass matrix $(\overline{A .9})$ by means of the angle $\overline{\theta_{t}}$, leading to the mass eigenstates $\bar{m}_{\widetilde{t}}$ and $\bar{m}_{\widetilde{T}}$. For $m_{Q} \gg T$ :

$$
\sin \theta_{t} \simeq \sin \bar{\theta}_{t} \simeq \frac{m_{t} \widetilde{A}_{t}}{m_{Q}^{2}}
$$

We can now expand the effective potential as a sum

$$
V(\phi, T)=V_{0}+V_{1}+V_{2}+\cdots
$$

where $V_{n}$ indicates the $n$-th loop potential in the resummed theory at finite temperature.

The tree-level potential can be written as:

$$
V_{0}=-\frac{1}{2} m^{2}(\bar{\mu}) \phi^{2}+\frac{1}{32}\left(g^{2}+g^{\prime 2}\right) \cos ^{2} 2 \beta \phi^{4}
$$

where

$$
m^{2}(\bar{\mu})=\frac{1}{2} m_{Z}^{2}(v) \cos ^{2} 2 \beta+\sum_{i} \frac{n_{i}}{16 \pi^{2}} m_{i}^{2}(v) \frac{d m_{i}^{2}(v)}{d v^{2}}\left[\log \left(m_{i}^{2}(v) / \bar{\mu}^{2}\right)+\frac{1}{2}-C_{i}\right]
$$


where $i=W, Z, h, \chi, t, \widetilde{t}, \widetilde{T}$, the constants $C_{i}$ are $C_{i}=5 / 6\left(C_{i}=3 / 2\right)$ for gauge bosons (scalar bosons and fermions), and the VEV of the Higgs field is normalized to $v=246$ $\mathrm{GeV}$.

We will perform daisy resummation on the $n=0$ modes of the longitudinal components of the gauge bosons $W_{L}, Z_{L}, \gamma_{L}$ and for all modes of the bosons $h, \chi$ and the light stop $\tilde{t}$. No resummation is performed on fermions (i.e. the top quark) or for heavy bosons which decouple from the thermal bath (in particular the heavy stop $\widetilde{T}$ ). With the above prescription we can write the one-loop effective potential $V_{1}$ as:

$$
V_{1}=\sum_{i} \frac{n_{i}}{64 \pi^{2}} M_{i}^{4}\left(\log \frac{M_{i}^{2}}{\bar{\mu}^{2}}-C_{i}\right)+\sum_{i} \frac{n_{i}}{2 \pi^{2}} J^{(i)} T^{4}
$$

where, as said before, for the analysis of the phase transition, we shall take $\bar{\mu}=T$. In the above, the sum extends over $i=W_{L}, Z_{L}, \gamma_{L}, W_{T}, Z_{T}, t, h, \chi, \widetilde{t}, \widetilde{T}$, and the masses $M_{i}$ are defined by

$$
M_{i}=\left\{\begin{array}{l}
m_{i} \quad i=W_{L}, Z_{L}, \gamma_{L}, W_{T}, Z_{T}, t, \widetilde{T} \\
\bar{m}_{i} \quad i=h, \chi, \widetilde{t}
\end{array}\right.
$$

The thermal contributions $J^{(i)}$ are defined by

$$
J^{(i)}= \begin{cases}J_{B}\left(m_{i}^{2}\right)-\frac{\pi}{6}\left(\bar{m}_{i}^{3}-m_{i}^{3}\right) & i=W_{L}, Z_{L}, \gamma_{L} \\ J_{B}\left(m_{i}^{2}\right) & i=W_{T}, Z_{T}, \widetilde{T} \\ J_{B}\left(\bar{m}_{i}^{2}\right) & i=h, \chi, \widetilde{t} \\ J_{F}\left(m_{i}^{2}\right) & i=t,\end{cases}
$$

and the thermal integrals $J_{B, F}$ are:

$$
J_{B, F}\left(y^{2}\right)=\int_{0}^{\infty} d x x^{2} \log \left(1 \mp e^{-\sqrt{x^{2}+y^{2}}}\right) .
$$

The two-loop potential can be split into the $\mathrm{SM}\left(V_{2}^{\mathrm{SM}}\right)$ and the MSSM $\left(V_{2}^{\mathrm{MSSM}}\right)$ terms.

$$
V_{2}=V_{2}^{\mathrm{SM}}+V_{2}^{\mathrm{MSSM}}
$$

The value of $V_{2}^{\mathrm{SM}}$ has been computed in Ref. [35] where the full expression can be found. It is dominated by logarithmic terms that can be written as:

$$
\begin{aligned}
V_{2}^{\mathrm{SM}} & =\frac{g^{2}}{16 \pi^{2}} T^{2}\left[-3\left(\frac{3}{2} m_{W}^{2}+2 \Pi_{W}\right) \log \frac{m_{W}+2 \bar{m}_{W_{L}}}{3 T}-\frac{63}{8} m_{W}^{2} \log \frac{m_{W}}{T}\right. \\
& \left.+\frac{3}{4} m_{W}^{2} \log \frac{2 \bar{m}_{W_{L}}}{3 T}+\frac{3}{2} m_{W}^{2} \log \frac{2 m_{W}}{3 T}+3 \bar{m}_{W_{L}} m_{W}\right]
\end{aligned}
$$

where the approximation $g^{\prime}=0$ has been used. There are also non-logarithmic contributions involving the strong $g_{s}$ and Yukawa $h_{t}$ couplings which are less important for the phase transition. 
In the two-loop potential $V_{2}^{\mathrm{MSSM}}$ the strong and top Yukawa couplings are involved in logarithmic contributions. Therefore we have found to be an excellent approximation to keep $g_{s}$ and $h_{t}$, and to neglect the weak couplings $g$ and $g^{\prime}$. In this approximation the relevant diagrams that contribute to $V_{2}^{\mathrm{MSSM}}$ are of two kinds: sunset diagrams, labeled by $V_{X Y Z}$, where $X, Y$ and $Z$ are the propagating fields, and figure eight diagrams, labeled by $V_{X Y}$, with propagating $X$ and $Y$ fields. With this prescription $V_{2}^{\mathrm{MSSM}}$ can be decomposed as:

$$
V_{2}^{\mathrm{MSSM}}=V_{\widetilde{t} \widetilde{t g}}+V_{\widetilde{t} \widetilde{t h}}+V_{g \widetilde{t}}+V_{\widetilde{t h}}+V_{\widetilde{t \chi}}+V_{\widetilde{t} \widetilde{t}}
$$

where $g$ stands for gluons. The different contributions are given by:

$$
\begin{aligned}
V_{\widetilde{t} \tilde{t} g} & =-\frac{g_{s}^{2}}{4}\left(N_{c}^{2}-1\right) \mathcal{D}_{S S V}\left(\bar{m}_{\widetilde{t}}, \bar{m}_{\widetilde{t}}, 0\right) \\
V_{\widetilde{t} \widetilde{t} h} & =-\left(h_{t}^{2} \sin ^{2} \beta \frac{\phi}{\sqrt{2}}+h_{t} \widetilde{A}_{t} \sin \beta \sin \bar{\theta}_{t} \cos \bar{\theta}_{t}\right)^{2} N_{c} H\left(\bar{m}_{h}, \bar{m}_{\widetilde{t}}, \bar{m}_{\widetilde{t}}\right) T^{2} \\
V_{g \widetilde{t}} & =-\frac{g_{s}^{2}}{4}\left(N_{c}^{2}-1\right) \mathcal{D}_{S V}\left(\bar{m}_{\widetilde{t}}, 0\right) \\
V_{\widetilde{t} h} & =\frac{1}{2} h_{t}^{2} \sin ^{2} \beta N_{c} I\left(\bar{m}_{\widetilde{t}}\right) I\left(\bar{m}_{h}\right) \\
V_{\widetilde{t} \chi} & =\frac{3}{2} h_{t}^{2} \sin ^{2} \beta N_{c} I\left(\bar{m}_{\widetilde{t}}\right) I\left(\bar{m}_{\chi}\right) \\
V_{\widetilde{t} \widetilde{t}} & =\frac{g_{s}^{2}}{6} N_{c}\left(N_{c}+1\right) I^{2}\left(\bar{m}_{\widetilde{t}}\right)
\end{aligned}
$$

The functions involved in (A.21) are all defined in Ref. [35]. We can use them with the following prescriptions:

- Cancel all poles in $1 / \epsilon$, and $\iota_{\epsilon}$-terms, with counterterms.

- Take the limit $\epsilon \rightarrow 0$ and keep all finite contributions.

- Cancel all $c_{B}$ terms for scalar bosons with thermal counterterms.

- Linear terms as $M_{i} T^{3}$ which appear in figure-eight diagrams are canceled by thermal counterterms.

Following these rules, we obtain results which are in good agreement with those found in Ref. [22].

\section{Appendix B}

In this appendix we will specify the effective potential at finite temperature in the background field $U \equiv \widetilde{t}_{R}^{\alpha} u_{\alpha}$, where $u_{\alpha}$ is a constant unit vector in color space f $^{\text {, which }}$ breaks $S U_{c}(3)$ into $S U(2)$. We will proceed as in appendix A and present the result of

\footnotetext{
${ }^{4}$ Using the $S U_{c}(3)$ invariance the vector $u_{\alpha}$ can be chosen as $u_{\alpha}=(1,0,0)$.
} 
the two-loop calculation. We will neglect $g^{\prime}$ and keep the strong $g_{s}$ and top Yukawa $h_{t}$ couplings. In the following, we use $g^{\prime}$ to denote one of the gluon states appearing in the spectrum.

The states contributing to the effective potential [18] are the four gluons $g$ and the gluon $g^{\prime}$, five real squarks $\omega$ (would-be goldstones) and the real squark $\rho$, four light $\mathcal{H}$ and four heavy $\mathcal{Q}$ scalars, coming from the mixing between the SM Higgs doublet $H$ and the left-handed (third generation) squark doublet $\widetilde{q} \equiv \widetilde{q}^{\alpha} u_{\alpha}$, and two degenerate massive Dirac fermions $f$ coming from the mixing between the left-handed (third generation) fermion doublet $q_{L} \equiv q_{L}^{\alpha} u_{\alpha}$ and the higgsino [18, 30]. The masses of $g, g^{\prime}, \omega, \rho$ and $f$ are given by:

$$
\begin{aligned}
m_{g}^{2} & =\frac{1}{2} g_{s}^{2} U^{2} \\
m_{g^{\prime}}^{2} & =\frac{2}{3} g_{s}^{2} U^{2} \\
m_{\omega}^{2} & =m_{U}^{2}+\frac{1}{3} g_{s}^{2} U^{2} \\
m_{\rho}^{2} & =m_{U}^{2}+g_{s}^{2} U^{2} \\
m_{f}^{2} & =\mu^{2}+h_{t}^{2} U^{2}
\end{aligned}
$$

and their degrees of freedom are

$$
\begin{array}{rll}
n_{g_{L}}=4 & n_{g_{T}}=8 \\
n_{g_{L}^{\prime}}=1 & n_{g_{T}^{\prime}}=2 \\
n_{\omega}=5 & & n_{\rho}=1 \\
n_{f} & =-8 . &
\end{array}
$$

The scalars $(H, \widetilde{q})$, with degrees of freedom

$$
n_{H}=4 \quad n_{\widetilde{q}}=4
$$

have a squared mass matrix

$$
\mathcal{M}_{H, \widetilde{q}}^{2}=\left(\begin{array}{cc}
-\frac{1}{2} m_{h}^{2}+h_{t}^{2} \sin ^{2} \beta U^{2} & h_{t} \sin \beta U \widetilde{A}_{t} \\
h_{t} \sin \beta U \widetilde{A}_{t} & m_{Q}^{2}+h_{t}^{2} U^{2}
\end{array}\right) .
$$

After diagonalization of the matrix (B.4) by the rotation defined through the angle $\theta_{\mathcal{H}}$, defined as in (A.6), we obtain the light $\mathcal{H}$ and heavy $\mathcal{Q}$ eigenstates and the corresponding eigenvalues, $m_{\mathcal{H}}^{2}$ and $m_{\mathcal{Q}}^{2}$.

Using the self-energies (A.7), and the gluon self-energy

$$
\Pi_{g}=\frac{8}{3} g_{s}^{2} T^{2}
$$

we can define the thermal masses for the longitudinal components of gauge bosons $\left(g_{L}\right.$, $\left.g_{L}^{\prime}\right)$ and for all scalar bosons $(\omega, \rho, \mathcal{H}, \mathcal{Q})$ as:

$$
\bar{m}_{g_{L}}^{2}=m_{g_{L}}^{2}+\Pi_{g}
$$




$$
\begin{aligned}
\bar{m}_{g_{L}^{\prime}}^{2} & =m_{g_{L}^{\prime}}^{2}+\Pi_{g} \\
\bar{m}_{\omega}^{2} & =m_{\omega}^{2}+\Pi_{\widetilde{t}_{R}} \\
\bar{m}_{\rho}^{2} & =m_{\rho}^{2}+\Pi_{\widetilde{t}_{R}}
\end{aligned}
$$

while $\bar{m}_{\mathcal{H}}^{2}$ and $\bar{m}_{\mathcal{Q}}^{2}$ are the eigenvalues of the thermally corrected squared mass matrix

$$
\overline{\mathcal{M}}_{H, \widetilde{q}}^{2}=\left(\begin{array}{cc}
-\frac{1}{2} m_{h}^{2}+h_{t}^{2} \sin ^{2} \beta U^{2}+\Pi_{h} & h_{t} \sin \beta U \widetilde{A}_{t} \\
h_{t} \sin \beta U \widetilde{A}_{t} & m_{Q}^{2}+h_{t}^{2} U^{2}+\Pi_{\widetilde{t}_{L}}
\end{array}\right) .
$$

diagonalized by the rotation $\bar{\theta}_{\mathcal{H}}$. For $m_{Q} \gg T$ :

$$
\sin \theta_{\mathcal{H}} \simeq \sin \bar{\theta}_{\mathcal{H}} \simeq \frac{h_{t} \sin \beta U \widetilde{A}_{t}}{m_{Q}^{2}}
$$

We will now expand the effective potential in a loop expansion as in (A.11),

$$
V(U, T)=V_{0}+V_{1}+V_{2}+\ldots
$$

The tree level potential can be written as:

$$
V_{0}=m_{U}^{2}(\bar{\mu}) U^{2}+\frac{1}{6} g_{s}^{2} U^{4}
$$

with

$$
m_{U}^{2}(\bar{\mu})=m_{U}^{2}-\sum_{i} \frac{n_{i}}{32 \pi^{2}} m_{i}^{2}(u) \frac{d m_{i}^{2}(u)}{d u^{2}}\left[\log \left(m_{i}^{2}(u) / \bar{\mu}^{2}\right)+\frac{1}{2}-C_{i}\right]
$$

where, as explained in Appendix A, we shall take $\bar{\mu}=T$. In the above, $i=g, g^{\prime}, \omega, \rho, f, \mathcal{H}, \mathcal{Q}$ and $u \equiv\langle U\rangle=\sqrt{-3 m_{U}^{2} / g_{s}^{2}}$.

We will perform, as in the case of appendix $\mathrm{A}$, resummation on the $n=0$ modes of the longitudinal components of the gauge bosons $g_{L}, g_{L}^{\prime}$ and for all modes of the bosons $\omega, \rho$ and the light scalar $\mathcal{H}$. No resummation is performed either on fermions $f$ or for the heavy scalar boson $\mathcal{Q}$, whose mass is dominated by the soft-term $m_{Q}$ and is decoupled from the thermal bath. We can then write the one-loop effective potential as in Eq. (A.14), where the sum extends over $i=g_{L}, g_{L}^{\prime}, g_{T} . g_{T}^{\prime}, f, \omega, \rho, \mathcal{H}, \mathcal{Q}$, and the masses $M_{i}$ are defined as:

$$
M_{i}=\left\{\begin{array}{l}
m_{i} \quad i=g_{L}, g_{L}^{\prime}, g_{T}, g_{T}^{\prime}, f, \mathcal{Q} \\
\bar{m}_{i} \quad i=\omega, \rho, \mathcal{H}
\end{array} .\right.
$$

The thermal contributions $J^{(i)}$ are defined

$$
J^{(i)}= \begin{cases}J_{B}\left(m_{i}^{2}\right)-\frac{\pi}{6}\left(\bar{m}_{i}^{3}-m_{i}^{3}\right) & i=g_{L}, g_{L}^{\prime}, \gamma_{L} \\ J_{B}\left(m_{i}^{2}\right) & i=g_{T}, g_{T}^{\prime}, \mathcal{Q} \\ J_{B}\left(\bar{m}_{i}^{2}\right) & i=\omega, \rho, \mathcal{H} \\ J_{F}\left(m_{i}^{2}\right) & i=f .\end{cases}
$$


The two loop diagrams that contribute to $V_{2}$ are, as in appendix A, of two kinds: sunset diagrams labeled by $V_{X Y Z}$, where $X, Y$ and $Z$ are propagating fields, and figure eight diagrams labeled by $V_{X Y}$, with propagating $X$ and $Y$ fields. In the following we will denote: $G \equiv\left(g, g^{\prime}\right)$ and $\tilde{t} \equiv(\omega, \rho), \eta$ being the ghost fields. The relevant contributions to $V_{2}$ are then (see also Ref. [30]):

$$
\begin{aligned}
& V_{G G G}=-g_{s}^{2} \frac{N_{c}}{4}\left[\left(N_{c}-2\right) \mathcal{D}_{V V V}\left(m_{g}, m_{g}, 0\right)+\mathcal{D}_{V V V}\left(m_{g}, m_{g}, m_{g^{\prime}}\right)\right] \\
& V_{\eta \eta G}=-g_{s}^{2} \frac{N_{c}}{2}\left[2\left(N_{c}-1\right) \mathcal{D}_{\eta \eta V}\left(0,0, m_{g}\right)+\mathcal{D}_{\eta \eta V}\left(0,0, m_{g^{\prime}}\right)\right] \\
& V_{\widetilde{t} \widetilde{t} G}=-\frac{g_{s}^{2}}{4}\left[\left(N_{c}-1\right) \mathcal{D}_{S S V}\left(\bar{m}_{\omega}, \bar{m}_{\omega}, m_{g}\right)+\left(N_{c}-1\right) \mathcal{D}_{S S V}\left(\bar{m}_{\omega}, \bar{m}_{\rho}, m_{g}\right)\right. \\
& +\frac{N_{c}-1}{N_{c}} \mathcal{D}_{S S V}\left(\bar{m}_{\omega}, \bar{m}_{\rho}, m_{g^{\prime}}\right)+\frac{1}{N_{c}} \mathcal{D}_{S S V}\left(\bar{m}_{\omega}, \bar{m}_{\omega}, m_{g^{\prime}}\right) \\
& \left.+N_{c}\left(N_{c}-2\right) \mathcal{D}_{S S V}\left(\bar{m}_{\omega}, \bar{m}_{\omega}, 0\right)\right] \\
& V_{\widetilde{t} G G}=-g_{s}^{2} \frac{m_{g}^{2}}{8}\left[\left(N_{c}-1\right) \mathcal{D}_{S V V}\left(\bar{m}_{\rho}, m_{g}, m_{g}\right)+2 \frac{\left(N_{c}-1\right)^{2}}{N_{c}^{2}} \mathcal{D}_{S V V}\left(\bar{m}_{\rho}, m_{g^{\prime}}, m_{g^{\prime}}\right)\right. \\
& \left.+\frac{\left(N_{c}-2\right)^{2}}{N_{c}} \mathcal{D}_{S V V}\left(\bar{m}_{\omega}, m_{g}, m_{g^{\prime}}\right)+N_{c}\left(N_{c}-2\right) \mathcal{D}_{S V V}\left(\bar{m}_{\omega}, m_{g}, 0\right)\right] \\
& V_{G G}=-g_{s}^{2} \frac{N_{c}}{8}\left[2\left(N_{c}-2\right) \mathcal{D}_{V V}\left(0, m_{g}\right)+2 \mathcal{D}_{V V}\left(m_{g}, m_{g^{\prime}}\right)+\left(N_{c}-1\right) \mathcal{D}_{V V}\left(m_{g}, m_{g}\right)\right] \\
& V_{\widetilde{t} G}=-\frac{g_{s}^{2}}{8}\left\{\left(N_{c}-1\right)\left[3 \mathcal{D}_{S V}\left(\bar{m}_{\omega}, m_{g}\right)+\mathcal{D}_{S V}\left(\bar{m}_{\rho}, m_{g}\right)\right]\right. \\
& +\frac{1}{N_{c}}\left[\left(N_{c}+1\right) \mathcal{D}_{S V}\left(\bar{m}_{\omega}, m_{g^{\prime}}\right)+\left(N_{c}-1\right) \mathcal{D}_{S V}\left(\bar{m}_{\rho}, m_{g^{\prime}}\right)\right] \\
& \left.+2 N_{c}\left(N_{c}-2\right) \mathcal{D}_{S V}\left(\bar{m}_{\omega}, 0\right)\right\} \\
& V_{\widetilde{t} \widetilde{t} \tilde{t}}=-\frac{g_{s}^{4}}{18}\left[3 H\left(\bar{m}_{\rho}, \bar{m}_{\rho}, \bar{m}_{\rho}\right)+\left(2 N_{c}-1\right) H\left(\bar{m}_{\rho}, \bar{m}_{\omega}, \bar{m}_{\omega}\right)\right] T^{2} U^{2} \\
& V_{\widetilde{t} \mathcal{H}}=-2\left[h_{t}^{2} \sin ^{2} \beta U+h_{t} \widetilde{A}_{t} \sin \beta \sin \bar{\theta}_{\mathcal{H}} \cos \bar{\theta}_{\mathcal{H}}\right]^{2} H\left(\bar{m}_{\omega}, \bar{m}_{\mathcal{H}}, \bar{m}_{\mathcal{H}}\right) T^{2} \\
& V_{\widetilde{t} \widetilde{t}}=\frac{1}{24} g_{s}^{2}\left[3 I^{2}\left(\bar{m}_{\rho}\right)+\left(4 N_{c}-2\right) I\left(\bar{m}_{\rho}\right) I\left(\bar{m}_{\omega}\right)+\left(4 N_{c}^{2}-1\right) I^{2}\left(\bar{m}_{\omega}\right)\right] \\
& V_{\widetilde{t} \mathcal{H}}=h_{t}^{2} \sin ^{2} \beta \cos ^{2} \bar{\theta}_{\mathcal{H}} I\left(\bar{m}_{\mathcal{H}}\right)\left[I\left(\bar{m}_{\rho}\right)+\left(2 N_{c}-1\right) I\left(\bar{m}_{\omega}\right)\right]
\end{aligned}
$$

where all functions involved in (B.14) are defined in Ref. [35] and cancellation of poles in $1 / \epsilon$, and $\iota_{\epsilon}$ and $c_{B}$ terms, as well as linear terms in $M_{i} T^{3}$, is achieved as in the case of appendix A against counterterms and thermal counterterms. 


\section{References}

[1] A.D. Sakharov, JETP Lett. 91B (1967) 24

[2] G. t'Hooft, Phys. Rev. Lett. 37 (1976) 8; Phys. Rev. D14 (1976) 3432

[3] P. Arnold and L. McLerran, Phys. Rev. D36 (1987) 581; and D37 (1988) 1020; S.Yu Khlebnikov and M.E. Shaposhnikov, Nucl. Phys. B308 (1988) 885; F.R. Klinkhamer and N.S. Manton, Phys. Rev. D30 (1984) 2212; B. Kastening, R.D. Peccei and X. Zhang, Phys. Lett. B266 (1991) 413; L. Carson, Xu Li, L. McLerran and R.-T. Wang, Phys. Rev. D42 (1990) 2127; M. Dine, P. Huet and R. Singleton Jr., Nucl. Phys. B375 (1992) 625

[4] For recent reviews, see: A.G. Cohen, D.B. Kaplan and A.E. Nelson, Annu. Rev. Nucl. Part. Sci. 43 (1993) 27; M. Quirós, Helv. Phys. Acta 67 (1994) 451; V.A. Rubakov and M.E. Shaposhnikov, Phys. Usp. 39 (1996) 461-502 [hep$\mathrm{ph} / 9603208$

[5] M. Shaposhnikov, JETP Lett. 44 (1986) 465; Nucl. Phys. B287 (1987) 757 and B299 (1988) 797

[6] G.W. Anderson and L.J. Hall, Phys. Rev. D45 (1992) 2685.

[7] M.E. Carrington, Phys. Rev. D45 (1992) 2933; M. Dine, R.G. Leigh, P. Huet, A. Linde and D. Linde, Phys. Lett. B283 (1992) 319; Phys. Rev. D46 (1992) 550; P. Arnold, Phys. Rev. D46 (1992) 2628; J.R. Espinosa, M. Quirós and F. Zwirner, Phys. Lett. B314 (1993) 206; W. Buchmüller, Z. Fodor, T. Helbig and D. Walliser, Ann. Phys. 234 (1994) 260

[8] J. Bagnasco and M. Dine, Phys. Lett. B303 (1993) 308; P. Arnold and O. Espinosa, Phys. Rev. D47 (1993) 3546; Z. Fodor and A. Hebecker, Nucl. Phys. B432 (1994) 127

[9] K. Kajantie, K. Rummukainen and M.E. Shaposhnikov, Nucl. Phys. B407 (1993) 356; Z. Fodor, J. Hein, K. Jansen, A. Jaster and I. Montvay, Nucl. Phys. B439 (1995) 147; K. Kajantie, M. Laine, K. Rummukainen and M.E. Shaposhnikov, Nucl. Phys. B466 (1996) 189; K. Jansen, Nucl. Phys. (Proc. Supl.) B47 (1996) 196 [hep-lat/9509018]. For an alternative approach, see: B. Bergerhoff and C. Wetterich, Nucl. Phys. B440 (1995) 171 and references therein

[10] G.R. Farrar and M.E. Shaposhnikov, Phys. Rev. Lett. 70 (1993) 2833 and (E): 71 (1993) 210; M.B. Gavela. P. Hernández, J. Orloff, O. Pène and C. Quimbay, Mod. Phys. Lett. 9 (1994) 795; Nucl. Phys. B430 (1994) 382; P. Huet and E. Sather, Phys. Rev. D51 (1995) 379

[11] M. Dine, P. Huet, R. Singleton Jr. and L. Susskind, Phys. Lett. B257 (1991) 351; A. Cohen and A.E. Nelson, Phys. Lett. B297 (1992) 111; P. Huet and A.E. Nelson, Phys. Lett. B355 (1995) 229; Phys. Rev. D53 (1996) 
[12] Y. Okada, M. Yamaguchi and T. Yanagida, Prog. Theor. Phys. 85 (1991) 1; H.E. Haber and R. Hempfling, Phys. Rev. Lett. 66 (1991) 1815

[13] J. Ellis, G. Ridolfi and F. Zwirner and B257 (1991) 83; Phys. Lett. B262 (1991) 477; A. Brignole, J. Ellis, G. Ridolfi and F. Zwirner, Phys. Lett. B271 (1992) 123; A. Brignole, Phys. Lett. B281 (1992) 284

[14] J.A. Casas, J.R. Espinosa, M. Quirós and A. Riotto, Nucl. Phys. B436 (1995) 3; M. Carena, J.R. Espinosa, M. Quirós and C.E.M. Wagner, Phys. Lett. B335 (1995) 209; M. Carena, M. Quirós and C.E.M. Wagner, Nucl. Phys. B461 (1996) 407; H. Haber, R. Hempfling and H. Hoang, Z. Phys. C75 (1997) 539; M. Carena, P. Zerwas and the Higgs Physics Working Group, in Vol. 1 of Physics at LEP2, G. Altarelli, T. Sjöstrand and F. Zwirner, eds., Report CERN 96-01, Geneva (1996)

[15] G.F. Giudice, Phys. Rev. D45 (1992) 3177; S. Myint, Phys. Lett. B287 (1992) 325

[16] J.R. Espinosa, M. Quirós and F. Zwirner, Phys. Lett. B307 (1993) 106

[17] A. Brignole, J.R. Espinosa, M. Quirós and F. Zwirner, Phys. Lett. B324 (1994) 181

[18] M. Carena, M. Quiros and C.E.M. Wagner, Phys. Lett. B380 (1996) 81

[19] D. Delepine, J.M. Gerard, R. Gonzalez Felipe and J. Weyers, Phys. Lett. B386 (1996) 183

[20] J. Cline and K. Kainulainen, Nucl. Phys. B482 (1996) 73; preprint MCGILL-97-7, hep-ph/9705201

[21] M. Laine, Nucl. Phys. B481 (1996) 43; M. Losada, Phys. Rev. D56 (1997) 2893; preprint [hep-ph/9612337]; G. Farrar and M. Losada, Phys. Lett. B406 (1997) 60

[22] J.R. Espinosa, Nucl. Phys. B475 (1996) 273

[23] B. de Carlos and J.R. Espinosa, preprint SUX-TH-97-005 hep-ph/9703212

[24] M. Carena, M. Quiros, A. Riotto, I. Vilja and C.E.M. Wagner, preprint CERNTH/96-242, hep-ph/9702409, to be published in Nucl. Phys. B

[25] J. Cline, M. Joyce and K. Kainulainen, preprint MCGILL-97-26, hep-ph/9708393

[26] T. Multamaki, I. Vilja, preprint TURKU-FL-P26-97, hep-ph/9705469

[27] A. Riotto, preprint OUTP-97-43-P, hep-ph/9709286

[28] M. Worah, preprint SLAC-PUB-7417, hep-ph/9702423

[29] M. Carena and C.E.M. Wagner, preprint FERMILAB-PUB-97-095-T, hepph/9704347], to appear in 'Perspectives on Higgs Physics II', ed. G. Kane, World Scientific, Singapore, 1997 
[30] D. Bodeker, P. John, M. Laine and M.G. Schmidt, Nucl. Phys. B497 (1997) 387

[31] K. Jansen, in Ref. [9]

[32] M. Carena, D. Choudhuri, S. Raychaudhury and C.E.M. Wagner, preprint CERNTH/97-176, hep-ph/9707458, to be published in Phys. Lett. B

[33] See, for example, P. Chankowki and S. Pokorski, preprint SCIPP-97-19, [hepph/9707497], to appear in 'Perspectives in supersymmetry', ed. G. Kane, World Scientific, Singapore, 1997

[34] M. Carena and C.E.M. Wagner, Nucl. Phys. B452 (1995) 45

[35] P. Arnold and O. Espinosa, in Ref. [8]

[36] P. Janot, talk given at the International Europhysics Conference on High Energy Physics, Jerusalem, August 1997.

[37] S. Bertolini, F. Borzumati, A. Masiero and G. Ridolfi, Nucl. Phys. B353 (1991) 591; R. Barbieri and G. Giudice, Phys. Lett. B309 (1993) 86.

[38] C. Greub, T. Hurth and D. Wyler, Phys. Lett. B380 (1996) 385; Phys. Rev. D54 (1996) 3350; K. Chetyrkin, M. Misiak and M. Münz, hep-ph/9612313; K. Adel and Y.P. Yao, Phys. Rev. D49 (1994) 4945; C. Greub and T. Hurth [hep-ph/9703349]; A.J. Buras, A. Kwiatkowski and N. Pott hep-ph/9707482, P. Ciafaloni, A. Romanino, A. Strumia, hep-ph/9710312; M. Ciuchini, G. Degrassi, P. Gambino, G.F. Giudice [hep-ph/9710335.

[39] M.S. Alam et al. (CLEO Collaboration), Phys. Rev. Lett. 74 (1995) 2885. 

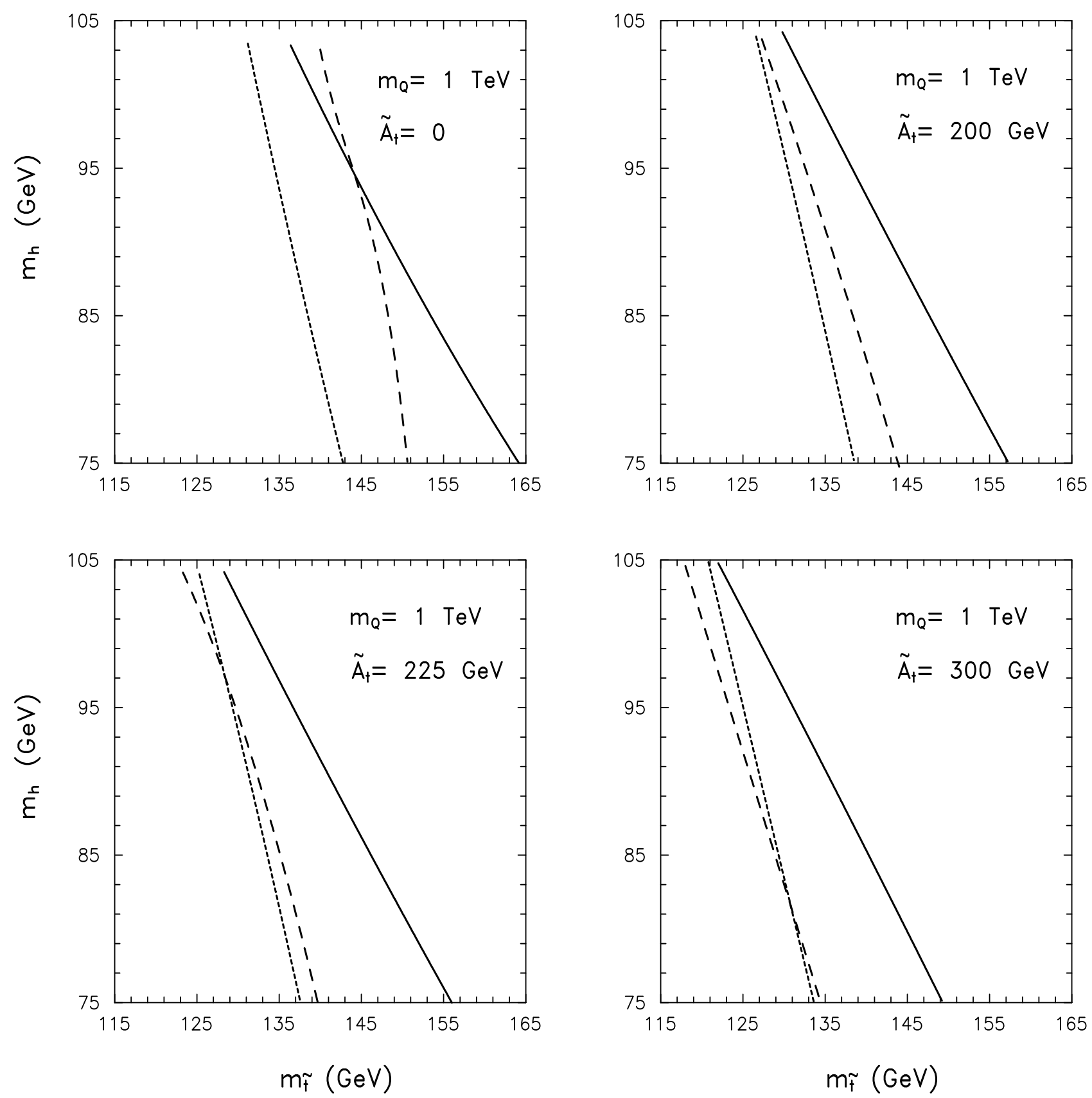

Figure 1: Values of $m_{h}, m_{\tilde{t}}$ for which $v\left(T_{c}\right) / T_{c}=1$ (solid line), $T_{c}^{U}=T_{c}$ (dashed line), $\widetilde{m}_{U}=\widetilde{m}_{U}^{c}$ (short-dashed line), for $m_{Q}=1 \mathrm{TeV}$ and different fixed values of $\widetilde{A}_{t}$. The region on the left of the solid line is consistent with a strongly first order phase transition. A two step phase transition may occur in the regions on the left of the dashed line, while on the left of the short-dashed line, the physical vacuum at $T=0$ becomes metastable. The region on the left of both the dashed and short-dashed lines leads to a stable color breaking vacuum state at zero temperature and is hence physically unacceptable. 


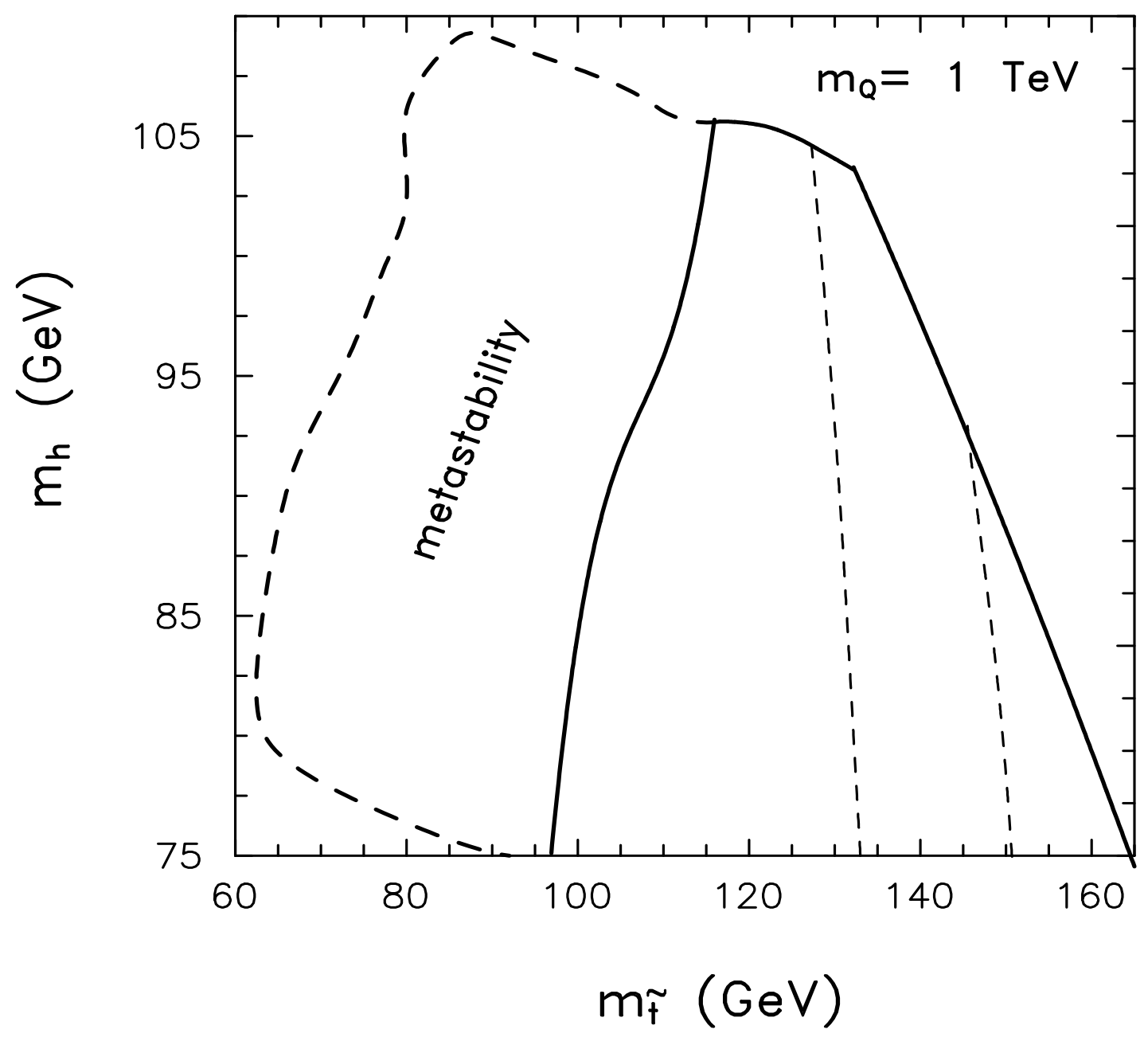

Figure 2: Region of the $m_{h}-m_{\widetilde{t}}$ parameter space for which a strongly first order phase transition takes place is shown within solid lines. The short-dashed lines demark the region for which a two-step phase transition may occur. The region on the right of the dashed line and left of the short-dashed may lead to a metastable vacuum state. 